\title{
"Working Towards Objective Detection Of Unknown Pathogens Via a Coordinated, Virtual Multiplex Microscopical Platform"
}

\author{
A. D. Hyatt \\ CSIRO, Division of Livestock Industries, Australian Animal Health Laboratory (AAHL), Private \\ bag 24, Geelong, Victoria, Australia.
}

For many years the mission of the AAHL diagnostic electron microscopy was to identify exotic viral incursions into Australia. However since the mid 1990s there has been the emergence of many diseases which have impacted on human, veterinary and wildlife health. These diseases are referred to as emerging infectious diseases which are defined as those that have recently increased in incidence, impact or geographic range; that are caused by pathogens that have recently evolved; that are newly discovered; or that have recently changed their clinical presentation. These diseases have become recognised as one of the most important threats to public, veterinary and wildlife health. Many of these new diseases are consequential to continued anthropogenic changes that serve to provide unique opportunities for the overlapping of naïve hosts with animals that have in many cases co-evolved with specific infectious organisms. As mans impact on the environment increases in the quest for more and more resources this recent trend will continue or even increase. It is therefore not surprising that combating emerging diseases is a key goal of public and veterinary health efforts both nationally and internationally.

More recently bio-terrorism has also emerged as a threat to human, veterinary health and trade. An essential ingredient to combating these emerging threats and minimizing their social and economic impacts is diagnostics. The mission of the AAHL diagnostic electron microscopy laboratory has therefore changed to include the detection of new and novel agents.

The classical methodology for detection of infectious agents includes negative contrast elelctron microscopy (generates data on surface structure), examination of ultrathin sections (generates information on cell morphogenesis where major steps in replication can be identified) and immuno-electron microscopy (generates information on antigenicity). Whilst these methods are critical to successful outcomes, 'diagnostics' must be performed with the knowledge that samples have been collected from optimal tissues. For example, ultra-thin sections should contain lesions representative of the disease. It is important the infectious organisms are observed in association with the lesions. In addition it is important at the light microscopy level that immunostaining is also associated with the lesion and the labeling pattern is consistent with the morphogenesis of the virus. For example, for paramyxoviruses the staining should be predominantly in the cytoplasm with the presence of cytoplasmic inclusion bodies and syncytia. It can not be over emphasized that when novel gents are being investigated light microscopy should be supported by electron microscopy. With this approach we can detect and identify the unknowns.

The major advantage of electron microscopy is that it was the first and is still the most robust multiplex diagnostic platform with the capacity for the simultaneous detection of micro and macro parasites. However, this technology is confronted with a major challenge namely to increase throughput. Increasing throughput in turn will be accompanied with other challenges including 
consolidation of experts in diagnostic virology (EM), bacteriology, mycology and parasitology, and the development and implementation of virtual, real-time national and international diagnostic microscopical networks. Such networks must have integrated middleware technology for the archiving of images and associated data, databases of the referred to experts and communication facilities including chat rooms and email facilities.

Progress in some of these areas has been made. For example, robotics are now being used in tomography to facilitate the imaging of large number of sections. Other workers are initiating studies defining critical parameters for the eventual automatic identification of viruses and others are working on developing software for real time consulting in diagnostic electron microscopy. Whilst the microscopical research community has initiated studies into developing an automated, objective, virtual, multiplex microscopical platform for the detection of unknown pathogens the commercial developers of software and columns appear, in the main, to be slow out-of-the-blocks. 